\title{
Final Report on HOLODEC 2 Technology Readiness Level
}

$\begin{array}{ll}\text { RA Shaw } & \text { SM Spuler } \\ \text { M Beals } & \text { N Black } \\ \text { JP Fugal } & \text { J Lu }\end{array}$

June 2012

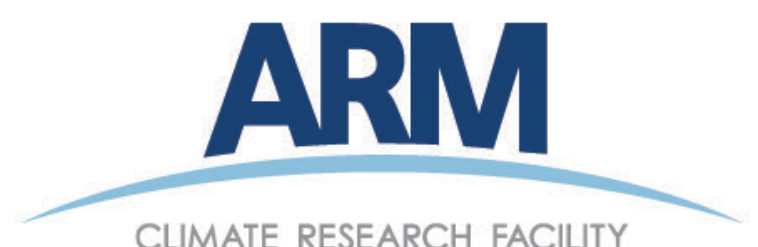




\section{DISCLAIMER}

This report was prepared as an account of work sponsored by the U.S. Government. Neither the United States nor any agency thereof, nor any of their employees, makes any warranty, express or implied, or assumes any legal liability or responsibility for the accuracy, completeness, or usefulness of any information, apparatus, product, or process disclosed, or represents that its use would not infringe privately owned rights. Reference herein to any specific commercial product, process, or service by trade name, trademark, manufacturer, or otherwise, does not necessarily constitute or imply its endorsement, recommendation, or favoring by the U.S. Government or any agency thereof. The views and opinions of authors expressed herein do not necessarily state or reflect those of the U.S. Government or any agency thereof. 


\title{
Final Report on HOLODEC 2 Technology Readiness Level
}

\author{
RA Shaw SM Spuler \\ M Beals N Black \\ JP Fugal J Lu
}

June 2012

Work supported by the U.S. Department of Energy,

Office of Science, Office of Biological and Environmental Research 


\section{Contents}

1.0 Overview of Current Technology Readiness Level..................................................................... 1

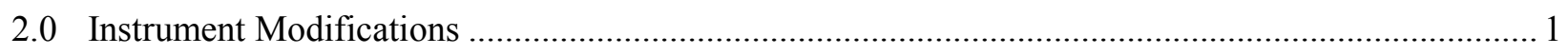

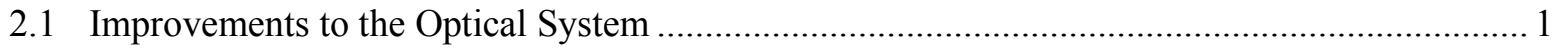

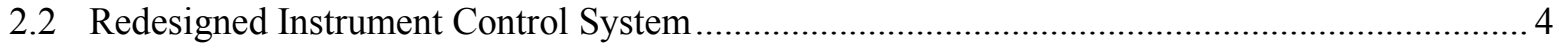

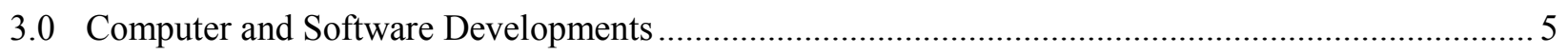

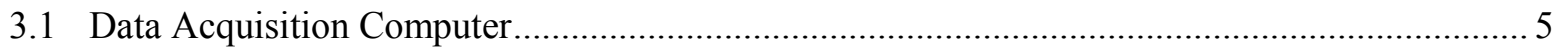

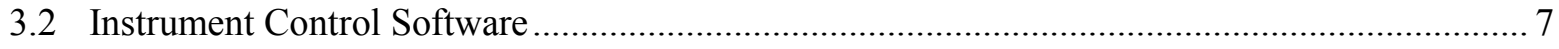

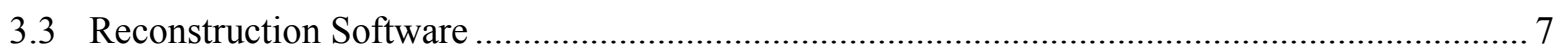

3.4 Reconstruction Methods for Improved Droplet Size Determination ........................................ 9

4.0 Data Sample from Instrument Deployment During IDEAS 2011 ................................................ 11 


\section{Figures}

Figure 1. The HOLODEC 2 instrument, far right, shown mounted on the NSF/NCAR C130 aircraft

Figure 2. Redesigned prism and window mounts and collimation optics mount. ..........................2

Figure 3. Alignment test setup with new mount to hold the beam expander spatial filter mount

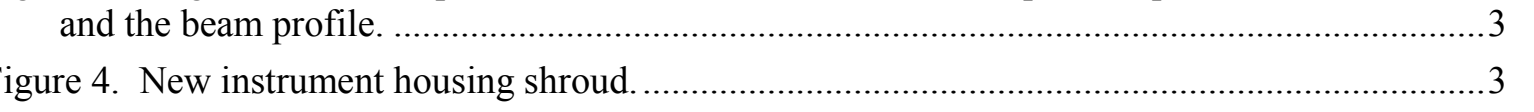
(1)

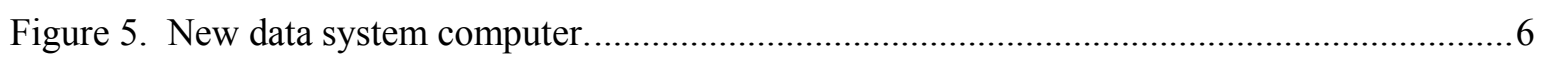

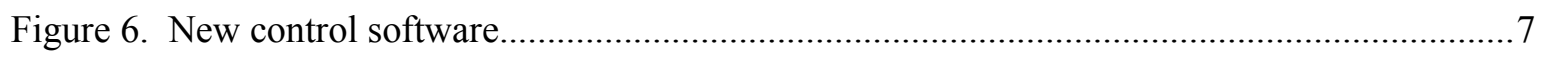

Figure 7. HoloViewer, the graphical front end of the hologram reconstruction and processing

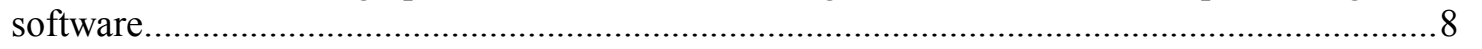

Figure 8. Conventional, unfiltered result of the axial intensity profile; the axial profile after applying the filter with a perfectly matched droplet diameter. .............................................. 10

Figure 9. Statistical results after reconstructing the 136 laboratory-recorded holograms and using the filter method to obtain the best particle size estimate. ....................................................... 11

Figure 10. Full HOLODEC sample volume showing droplet spatial locations and droplet diameter. The resulting size distribution is plotted to the right and is compared to one

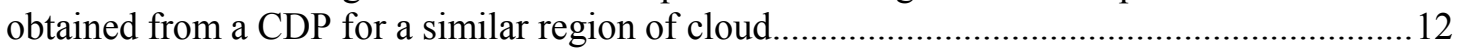

Figure 11. Reconstructed ice crystal images from HOLODEC ............................................... 12 


\subsection{Overview of Current Technology Readiness Level}

During the period of this project, the Holographic Detector for Clouds 2 (HOLODEC 2) instrument (Figure 1) has advanced from a laboratory-proven instrument with some initial field testing to a fully flight-tested instrument capable of providing useful cloud microphysics measurements. This can be summarized as "Technology Readiness Level 8: Technology is proven to work - Actual technology completed and qualified through test and demonstration." As part of this project, improvements and upgrades have been made to the optical system, the instrument power control system, the data acquisition computer, the instrument control software, the data reconstruction and analysis software, and some of the basic algorithms for estimating basic microphysical variables like droplet diameter. Near the end of the project, the instrument flew on several research flights as part of the IDEAS 2011 project, and a small sample of data from the project is included as an example. There is one caveat in the technology readiness level stated above: the upgrades to the instrument power system were made after the flight testing, so they are not fully field proven. We anticipate that there will be an opportunity to fly the instrument as part of the IDEAS project in fall 2012.

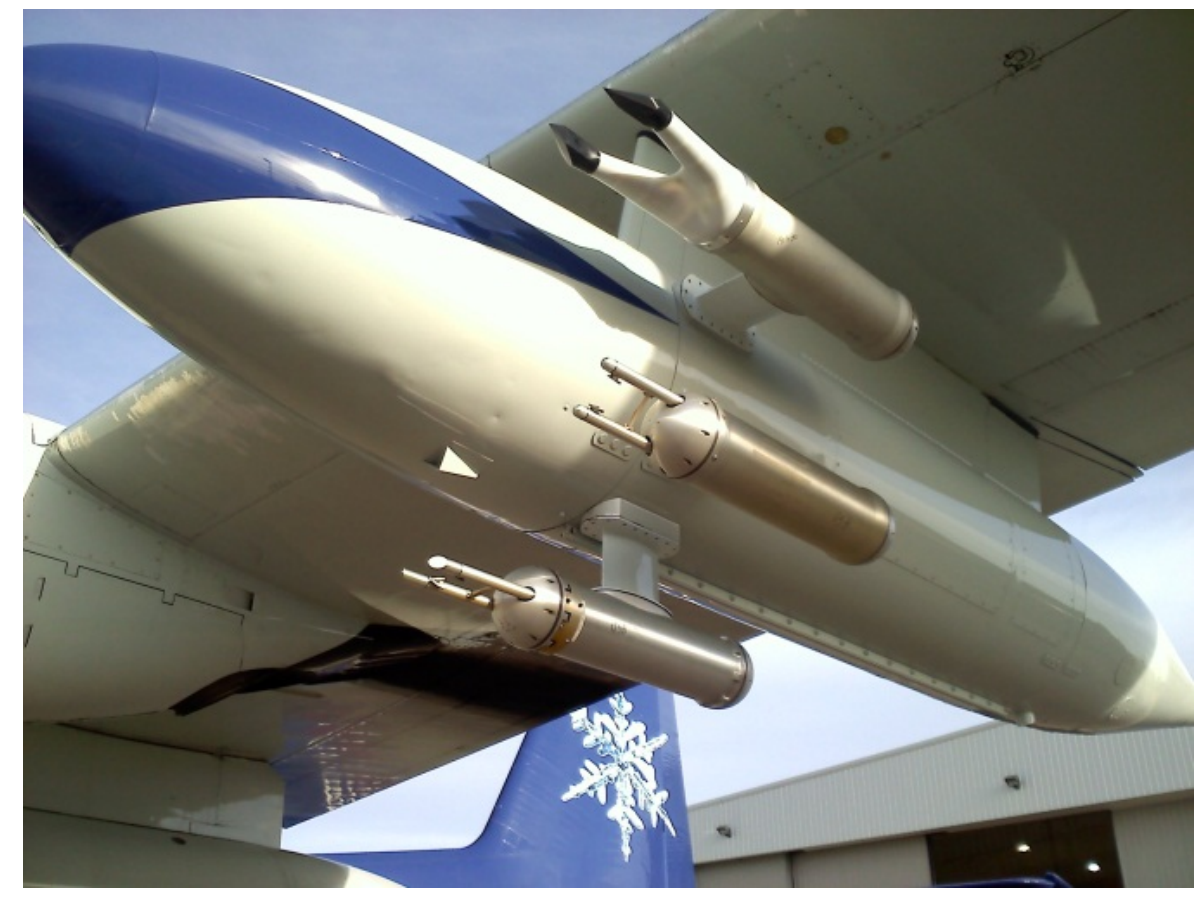

Figure 1. The HOLODEC 2 instrument, far right, shown mounted on the NSF/NCAR C130 aircraft.

\subsection{Instrument Modifications}

\subsection{Improvements to the Optical System}

The optical system of HOLODEC underwent a series of small upgrades that, when taken together, have led to a significant improvement in hologram quality (i.e., lower noise floor) and therefore in the particle measurement resolution. The specific upgrades are described below. 
Turning Optics: The turning optics (prisms, windows) at the ends of the probe arms were redesigned. The original design introduced anamorphic magnification and some minor aberrations that resulted in variable magnification with position. Although correctable via a resolution target calibration as a function of position, this was not optimal. New non-wedged windows and 81-degree prisms were designed and fabricated along with a new optical-mechanical window plate assembly. Resolution target tests were performed that demonstrated that the new optical design worked and meets the expected instrument performance as discussed in Spuler and Fugal (2011, "Design of an in-line, digital holographic imaging system for airborne measurement of clouds," Applied Optics.) Figure 2 shows the new prism and window mounts (the new collimation optics mount is in the upper right-hand corner).

Collimation Optics: The collimation optics mount was redesigned to improve beam collimation and weather sealing and to allow for easier removal (e.g., easier to replace in the field). The image below shows the new collimation optics mount in the upper right-hand corner.

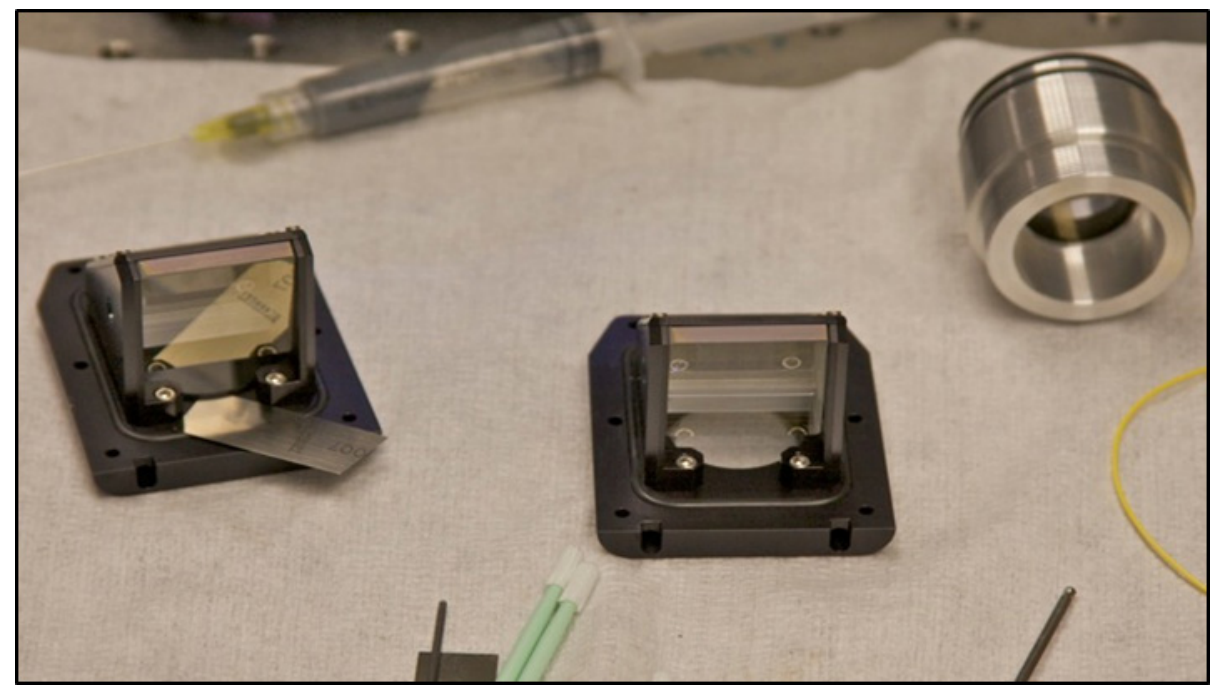

Figure 2. Redesigned prism and window mounts (left and center) and collimation optics mount (right).

Beam Profile: The laser beam uniformity was improved by adding a spatial filter. This upgrade enhances the ability to identify particles in the reconstructed holograms. The spatial filter is a $75-\mu \mathrm{m}$ diameter natural diamond aperture mounted in a 1 " $\mathrm{OD} \times 0.25$ " thick stainless steel housing. The expanding lens mount was redesigned to hold both the expanding lens and the spatial filter in a single mount. The mount attaches directly to the laser head. Figure 3 shows the alignment test setup with the new mount to hold the beam expander spatial filter mount and the beam profile (note this is prior to collimation optics). 

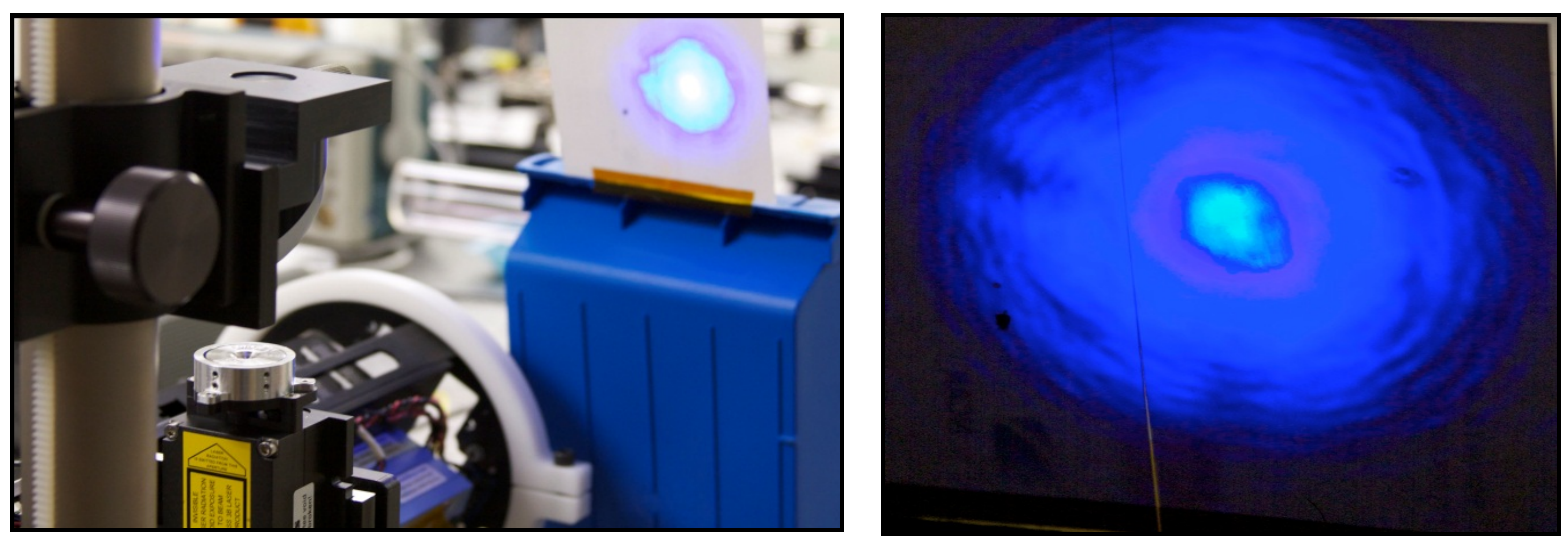

Figure 3. Alignment test setup with new mount to hold the beam expander spatial filter mount (left) and the beam profile (right).

Environmental Sealing: The environmental sealing of the probe tips was improved for more robust operation in harsh field conditions. This included sealing the set-screw holes with epoxy and modifying the outer diameter of the probe arms to allow for O-ring sealing.

Instrument Housing: A new shroud was fabricated from an engineered plastic: $30 \%$ glass-filled Noryl. The original nylon shroud material proved very difficult to install correctly when deployed in humid environments, as it would absorb water and change shape. The new shroud is dimensionally stable with significantly lower moisture absorption and thermal expansion. It will ease installation of the instrument in the future. A picture of the new shroud is shown in Figure 4.

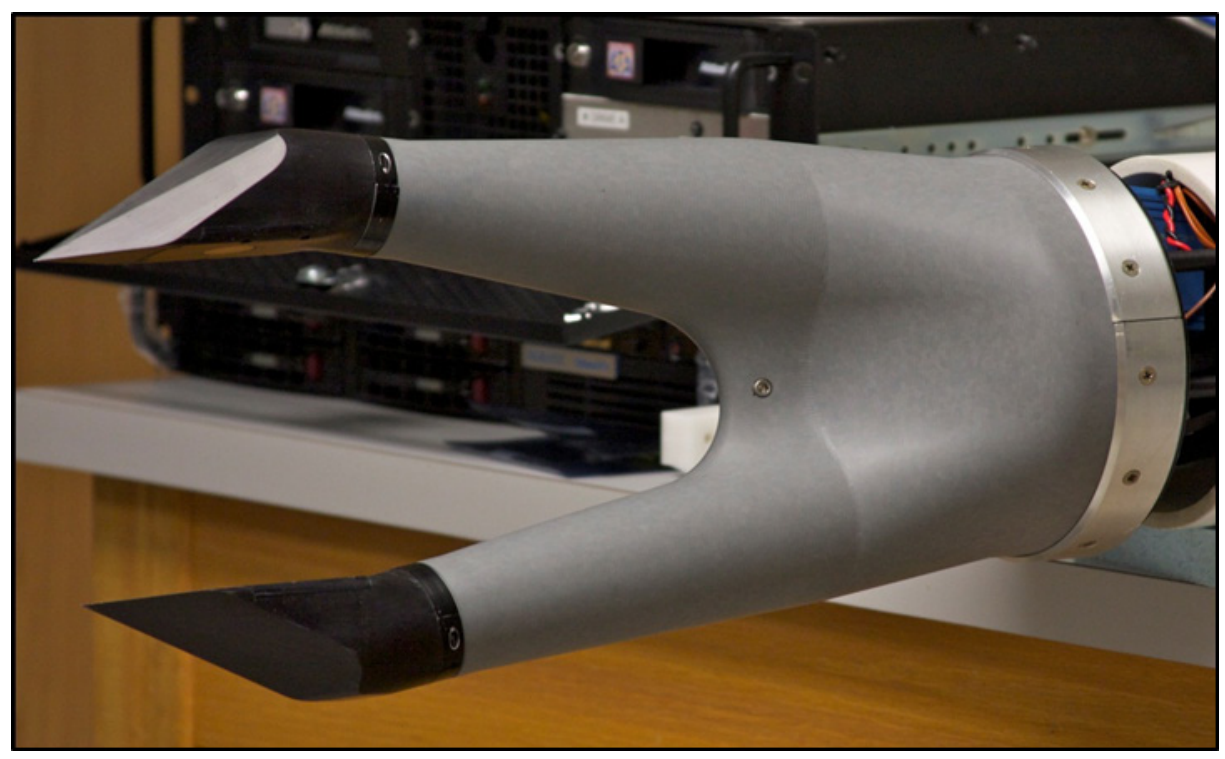

Figure 4. New instrument housing shroud.

Laser Head: A spare laser head was purchased, as it is a critical item with a long lead time. Also, a new power supply controller unit was tested that verified that the laser is capable of long-term operation without power fluctuations. 


\subsection{Redesigned Instrument Control System}

The instrument control system for HOLODEC has been thoroughly updated to allow for more automated functionality and for better integration into typical research aircraft data systems. The primary design goal in the upgrade was to make the instrument useable to a wider range of scientists and research aircraft. Specific system upgrades are described below.

Power System Upgrades: The upgrades involve two parts, the first being the 12-VDC power subsystem. The original design consisted of a single AC-DC converter supplying 12 VDC power to the laser, camera, and control system electronics. Power to individual components was controlled by power relays in the circuit. Two problems were identified with this arrangement: the peak current draw of the laser exceeded the capacity of the relays, causing damage and introducing undesirable behavior, and line noise from variable loads from the other components was potentially interfering with laser operation. To address these issues, the power supply was replaced with a unit with independent power modules, such that each $12-\mathrm{V}$ component is on an isolated supply. Switching is now performed at the PSU as well, creating smooth power transitions and improving power quality to all 12-VDC components. The second part of the upgrade was to the 120-VAC power system. The main 120-VAC power system supplies power for the heating subsystem and for the AC-DC converter used to power the 12-VDC subsystems. The original design made use of a simple clip-style fuse block for over-current protection, which may have contributed to power system failures in the field. The entire 120-VAC power distribution system was redesigned with mil-spec hardware to improve robustness and ease of field repair work.

Thermal Management: The first part of the upgrades to the thermal management system involves the heaters themselves. In the original system, only the laser, camera, and instrument tips were actively heated. It was observed that the laser control electronics were falling out of the standard operating temperature range, and the heater controllers began showing instability at temperatures observed during high altitude flight. To improve the stability of the control electronics, additional heaters are installed such that all parts of the probe are heated. The heaters installed to warm the main control electronics make use of a simple bi-metal thermostat such that their performance does not rely on the control electronics operating correctly. This is a safety measure to ensure that the control electronics remain at operating temperature as long as power is applied to the instrument.

The second part of the thermal management upgrades involves automated heater control.

The power relays used to switch power to the instrument subsystems were controlled manually by an operator in the aircraft in the prior design. During aircraft maintenance flights, however, there are typically no technicians or crew allowed on board so the instrument heaters would not be turned on. Without heat, the laser would get too cold, and the optics would go out of alignment. To address this situation, the relay control board is replaced with a custom-built FPGA-based logic control board. This board is programmed to automatically enable the heaters at power-up, ensuring that the instrument's sensitive electronics are always within operating temperature. The new control board is also programmed with a routine to check the current temperature of the electronics before enabling the heater circuits so that it can dynamically reprogram the heater controllers to limit the heating rate. This ensures that electronics are not heated too quickly in the event that power is lost and restored in flight (i.e., if the electronics cool well below their set point temperature). Finally, in addition to programmatic temperature control, non-resettable thermal fuses are installed in the heated components. These fuses are designed to interrupt the heater power circuit in the event they exceed a set temperature. These are added to protect 
against over-temp conditions if equipment were to fail and cause a heater to become permanently engaged.

Communication Subsystem: The first part of the upgrades to the communication subsystem involved consolidation of all components. The new control board used to control the heater controllers is also used to consolidate the communications with the instrument. The original system consisted of individual serial communication lines (one for each subsystem) running between the instrument and control computer inside the aircraft. In the new design, all communications are routed through the new communications board. This new arrangement:

- Creates a single, unified command interface for the instrument

- Improves robustness by reducing possible points of failure

- Simplifies in-wing wiring requirements, allowing the instrument to potentially fly on a wider range of platforms

- Allows for automated control by the control board logic.

The second part of the communication system upgrade involved addition of 10/100 Ethernet control. The new control board also implements communication by 10/100 Ethernet in addition to RS485. This is designed to increase the flexibility of the instrument and allow it to better integrate with typical onboard data systems for research aircraft (e.g., those used on the NCAR C-130 and G-V). This allows the aircraft data system to start up, shut down, and request status information from the instrument and allows the instrument to broadcast a stream of "housekeeping" data to the aircraft data system. This datastream maintains a permanent log of instrument temperatures any time the instrument flies. Ethernet-based communication is also becoming more popular for aircraft instrumentation, and this upgrade helps prepare the instrument for future missions.

\subsection{Computer and Software Developments}

\subsection{Data Acquisition Computer}

The original data acquisition computer consisted of a laptop mounted to a rackmount tray. This configuration required the laptop lid to be almost completely closed during operation, which led to potential problems with overheating. The laptop also lacked an internal Ethernet card with the specifications required by the probe's camera, the proper serial communication ports, and any removable hard drive bays, requiring all three components to be individually mounted external to the same tray. The many wires needed to connect the various components to the laptop were exposed and prone to being disconnected when the tray was pulled out to access the laptop. Of particular concern was the external ExpressCard Ethernet adapter, which when forcibly removed from the system while running would corrupt the driver and crash the operating system. This required special procedures to recover the system. 


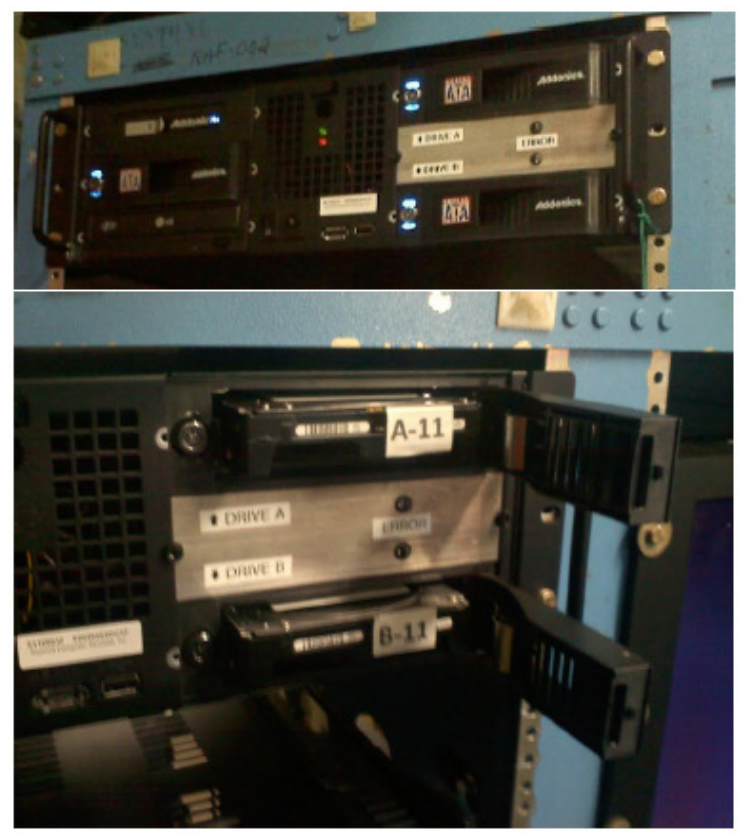

Figure 5. New data system computer.

The new data system computer, shown in Figure 5, was designed to address these specific weaknesses as well as to increase the system's failure tolerance and versatility. The key aspects of the new system are as follows:

Single Chassis: The chassis for the system is a standard 3U rackmount chassis, with all components needed to run the probe contained within. This reduces the number of external connections to a minimum and eliminates the need to pull the system out from the rack during operation, further reducing the stress on the connections.

Improved Cooling: The primary cause for the overheating with the laptop was that it was being used in a manner outside of its designed tolerances. The new system is being run in the orientation it was designed to operate in, allowing the cooling system to function at full capacity.

Fault Tolerance: The new system is configured with a solid state main system disk to reduce the likelihood of corruption from vibration or impact shock. The system also has a second, offline system disk onboard that can be used to boot the system in the event that the main drive becomes unbootable. Both disks are mounted in vibration-isolated, front-mount racks, allowing them to be easily exchanged for working discs in the field.

Data Discs: The HOLODEC 2 probe produces data at a rate of approximately $1 / 6 \mathrm{~TB}$ per hour. To handle these large data loads, standard SATA hard drives are used as removable storage. The original system used an external enclosure that held two 3.5" drives in a RAID1 configuration. The RAID1 is employed primarily for data redundancy after the drives leave the aircraft, but it also provides a small level of fault tolerance, as heavy impacts have been observed to cause a missed write in one drive that is quickly corrected by the RAID controller. The external enclosure of the old system was replaced with an internal hardware RAID controller and two front-mount drive racks mounted in the data acquisition system. The 
drive racks are vibration-isolated and do not require the drives to be mounted to special sleds. This simplifies the process of swapping out drives and reduces operational costs. The system can also be configured to use either 3.5" or 2.5" drives. The 2.5" drives cannot support flights in excess of 6 hours, but their reduced size and weight make for easer transport and storage.

\subsection{Instrument Control Software}

Communication and control of the instrument is achieved through four comm channels, which provide control and monitoring of the power distribution relays, the heater controllers, the laser controller, and eight channels of thermocouples. The heater controllers have factory-supplied software for configuration and monitoring, but control of the power system and monitoring of the thermocouples was performed through a TTY terminal that required the operator to be familiar with the specific command set for the components.

To simplify and unify the control of the probe, new software was developed in LabView that handles the control of the instrument under a single, simplified graphical interface. This interface allows an operator with minimal training to control all aspects of the system and to monitor datastreams as well as housekeeping data such as temperatures and component status. A photo showing the control software during a test flight on the NCAR C130 is shown in Figure 6.

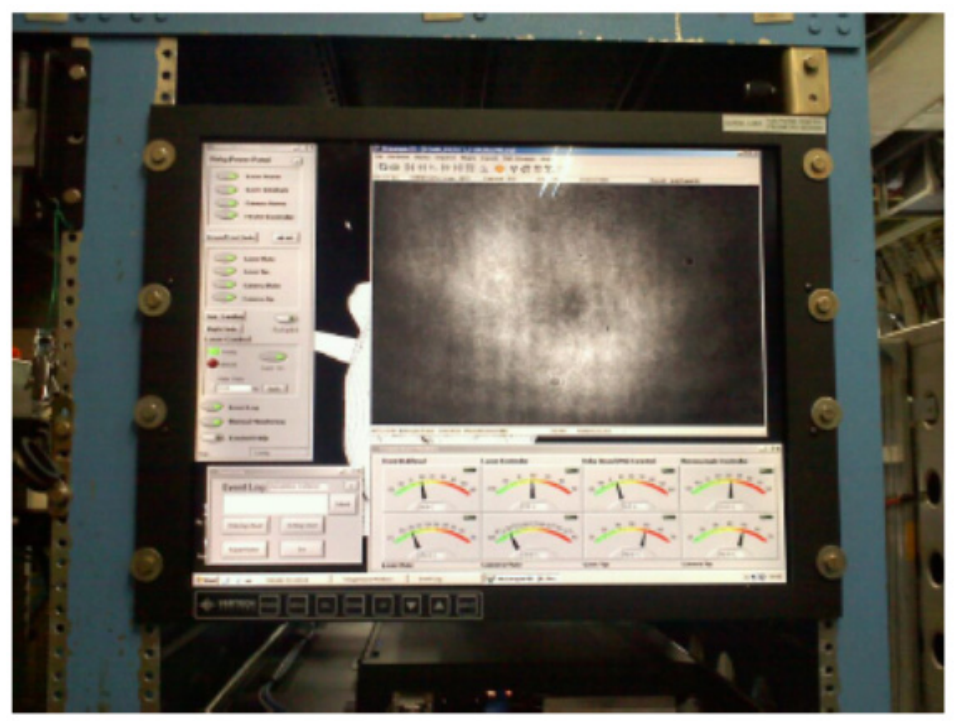

Figure 6. New control software.

\subsection{Reconstruction Software}

In addition to improving the probe to ensure that the highest quality of data is being recorded, the hologram reconstruction and processing software has undergone a major overhaul. The current version of the software is inspired by the "HoloViewer" project developed by Dr. Jacob Fugal for the first HOLODEC instrument. We have helped expand it into a suite of tools now known as "HoloSuite." HoloSuite allows a user to reconstruct and analyze holograms without having to understand intimate details of the reconstruction method, while at the same time remain flexible enough to appeal to more 
advanced users. This is accomplished by dividing the project into two parts: a collection of code-based tools and a graphical front end.

The code-based tools are based around three main processing objects that provide a modular framework to the reconstruction process. Key advances in the new code base include:

- GPU- and CPU-based reconstruction are handled seamlessly, including smart memory management to deal with holograms that are too large for GPU processing.

- Caching of data that does not change often, reducing redundant calculations and improving performance.

- Image input and output, including filtering, brightness/contrast enhancement (including an autocontrast feature), and threshold and amplitude/phase transformations (for complex valued images) are handled internally.

- User functions can be implemented as custom filters and can be inserted anywhere in the reconstruction chain.

- Settings, including reconstruction parameters, filtering schemes, and threshold settings, are preserved, allowing easy transfer between a graphical interface and automated processing code.
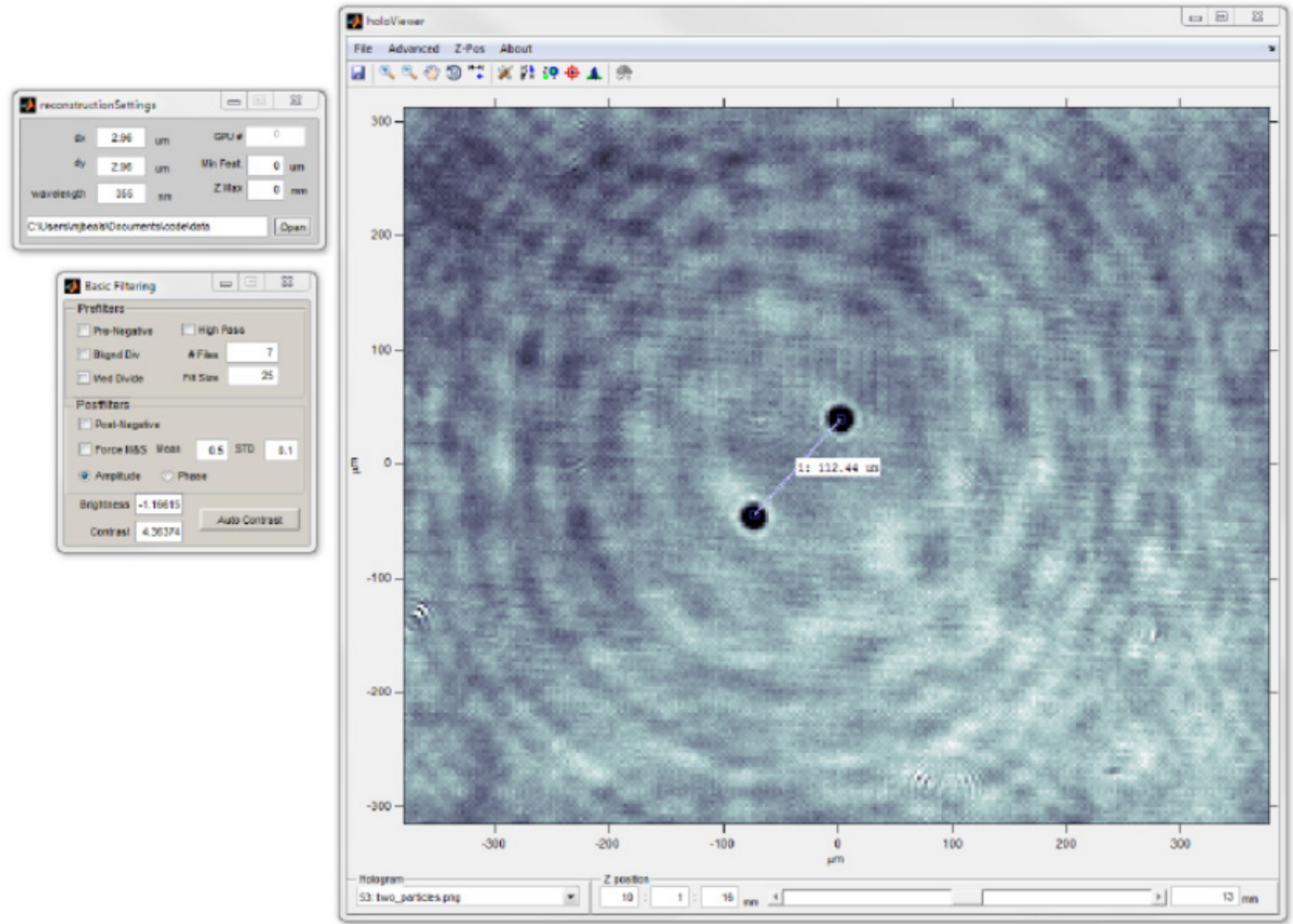

Figure 7. HoloViewer, the graphical front end of the hologram reconstruction and processing software.

Built upon this code base is the graphical front end, HoloViewer (shown in Figure 7). This program follows the format of its predecessor and allows a user to browse through holograms, both in time (hologram to hologram) and in depth position (using live reconstruction). With HoloViewer a user is also able to apply any number of built-in or custom filters, seeing the effect on the reconstructed hologram 
almost immediately. The software also allows the user to adjust the threshold parameters used by the automated reconstruction code and visualize how regions of the reconstruction are being classified as particles, as well as the effect that dilation will have on that region and how the computer will "see" the region when it computes the gradient for particle edge detection.

The final piece of software is the automated particle-finding routines. These routines are designed to reconstruct a holographic volume slice by slice, searching each slice for pixels exceeding the preset thresholds and stitching these selected pixels into volumetric particle groups. These groups of voxels (3D pixels) are further processed with a variety of techniques to determine the particle's size and shape, as well as where it comes into focus (yielding its depth position). A new algorithm that utilizes both amplitude and phase information has proven extremely efficient at identifying cloud droplets and rejecting noise.

\subsection{Reconstruction Methods for Improved Droplet Size Determination}

We have developed a method for greatly improving the accuracy of droplet size estimation from digital in-line holograms and have verified the improvement using simulated and experimental data. The method utilizes what we call a "sign-matched filter"; the Fourier-domain filter is equivalent to the sign operator on the angular spectrum of a preselected aperture function. The approach is easily implemented within the conventional hologram reconstruction method. When the aperture function has a diameter equal to that of a measured particle, there is a strong peak in the reconstructed field. A range of filters for different particle sizes is applied, and the maximum in the peak-intensity versus filter particle size gives the correct particle size. As implemented currently, conventional reconstruction and pixel counting is used to obtain coarse particle size information, and then the size estimate is refined through the filter method.

Furthermore, when the accurate size information is obtained, we simultaneously have obtained an improved estimate of the particle position along the optical axis (improved depth resolution). Finally, the method has proven to be robust to additive noise and can be applied to a wide range of particle sizes, even if those particles are in the same hologram.

This processing algorithm is now in the stage of being integrated into the HoloSuite set of software tools. Based on the initial simulation and laboratory tests, we anticipate that it will improve the size estimation for cloud droplet diameter by approximately a factor of five. This is a significant advance in the capability of the holographic measurement method and a paper describing the method has been published in Optics Express (Lu et al. 2012).

Figure 8 illustrates the effect of the filter on the axial intensity profile for a simulated particle (i.e., the intensity profile along the optical axis of the holographic system, through the center of a particle). The left panel shows the conventional, unfiltered result, and the right panel shows the axial profile after applying the filter with a perfectly matched droplet diameter, i.e., for filter diameter equal to the particle diameter of $56 \mu \mathrm{m}$. The sharp peak decays when the filter diameter does not match the droplet diameter. 
(a)

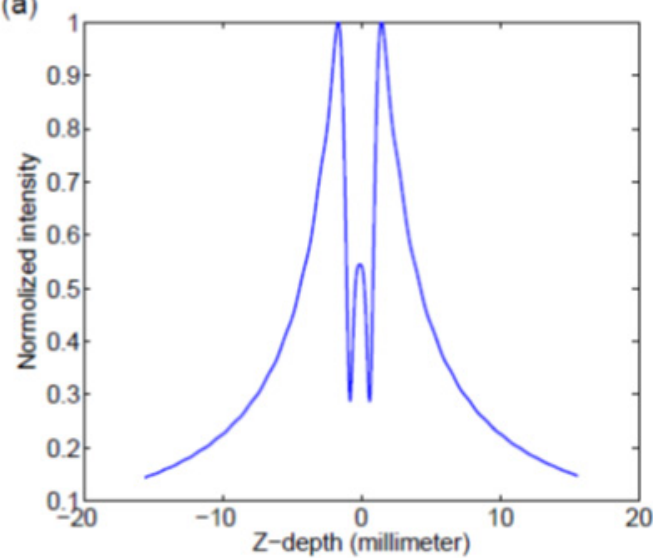

(b)

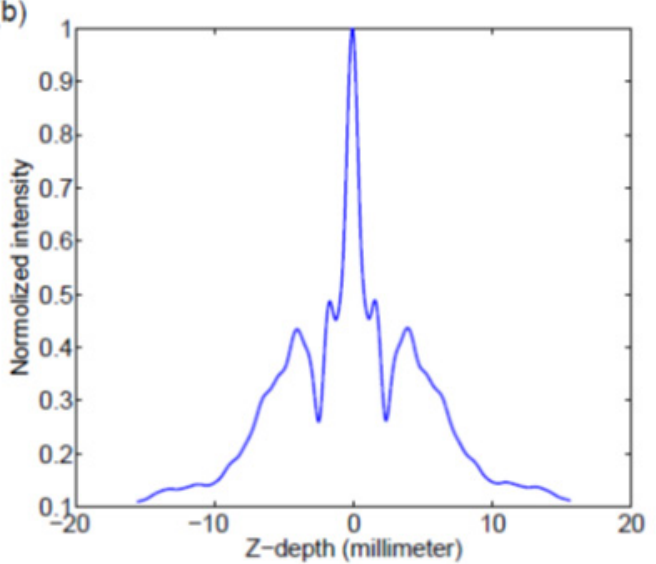

Figure 8. (Left panel) Conventional, unfiltered result of the axial intensity profile; (right panel) the axial profile after applying the filter with a perfectly matched droplet diameter.

To verify the effectiveness of this method, we also obtained analyzed holograms recorded in the laboratory. The optical system consists of a frequency doubled Nd:YLF laser $(\lambda=0.527 \mu \mathrm{m})$. The beam is passed through a beam expander with the addition of a pinhole for spatial filtering. The camera is an Illunis XMV-11000 with $4008 \times 2672$ pixels with $9-\mu \mathrm{m}$ pixel pitch and 12-bit output. Water droplets are injected by a piezoelectric injector that generates monodisperse droplets. The mean droplet diameter is $\overline{\mathrm{d}}$ $=56.1 \mu \mathrm{m}$ with an upper bound on the size variability of $\Delta \mathrm{d}=0.5 \mu \mathrm{m}$, as determined from a high resolution, telecentric microscope with a spatial resolution of $0.9 \mu \mathrm{m}$ (the diameter can be determined to better than the spatial resolution by considering the full area of the image). The experimental data consist of 136 holograms taken at a depth of $\mathrm{z}_{0}=531 \mathrm{~mm}$, with one particle taken from each hologram. The particles are taken at the same location in the hologram to avoid any possible evaporation effects. First, we used conventional reconstruction and pixel counting to obtain the coarse particle size and location. Then each particle in the hologram was processed using the sign-matched filtering method described here. We then repeated the reconstruction with filters corresponding to particle diameters in $0.2 \mu \mathrm{m}$ increments and searching for the axial intensity profile with the maximum peak. It shows that the peak is around 56 $\mu \mathrm{m}$.

The red histogram (Figure 9) shows the statistical results after reconstructing the 136 holograms and using the filter method to obtain the best particle size estimate. For comparison, the blue histogram shows the results from a direct reconstruction and pixel-counting method (pixel counting based on a global intensity threshold), which is the prior method for particle size determination with HOLODEC. When using pixel counting the mean particle diameter is $\overline{\mathrm{d}}=55.7 \mu \mathrm{m}$, and the standard deviation is $\sigma_{\mathrm{d}}=2.8 \mu \mathrm{m}$. This standard deviation is consistent with the uncertainty scaling with square root of pixel size. For the matched filtering method the mean size is $\overline{\mathrm{d}}=\mathrm{d}^{-}=56.0 \mu \mathrm{m}$, and the standard deviation is $\sigma_{\mathrm{d}}=0.23 \mu \mathrm{m}$. This standard deviation is consistent with the expected variability from the piezoelectric droplet generator. This clearly demonstrates the ability of the new method to greatly improve the precision of particle size estimation. 


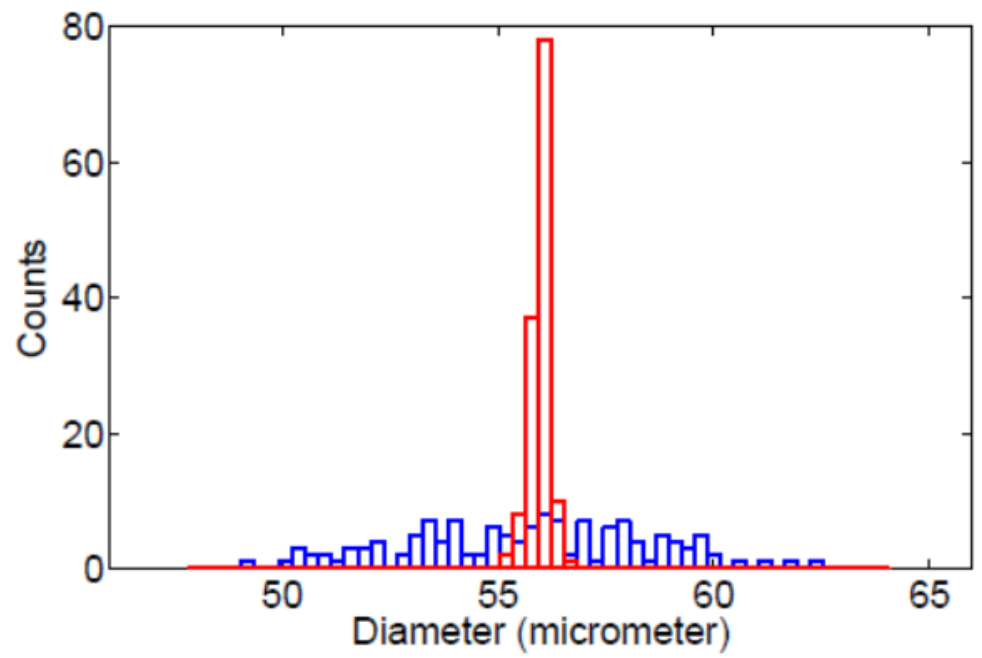

Figure 9. Statistical results after reconstructing the 136 laboratory-recorded holograms and using the filter method to obtain the best particle size estimate.

\subsection{Data Sample from Instrument Deployment During IDEAS 2011}

The improved HOLODEC instrument flew on the NCAR C130 in October 2011 as part of the Instrument Development and Education in the Airborne Sciences (IDEAS) project. This provided an ideal environment for instrument testing under realistic flight conditions, with plenty of time between flights for instrument modifications and data quality checks. During several research flights the HOLODEC sampled liquid and mixed-phase clouds, leading to a diverse data set that is just starting to be analyzed. A complete data description will be released separately, but here several images are included as illustrations of the instrument capabilities.

Figure 10 is a full HOLODEC sample volume showing droplet spatial locations and droplet diameter (via color coding). The resulting size distribution is plotted to the right and is compared to one obtained from a CDP for a similar region of cloud. The critical distinction, however, is that the full size distribution from HOLODEC is obtained from a localized cloud volume of approximately $15 \mathrm{~cm}^{3}$, whereas typical optical instruments like the CDP have to average over flight paths on the order of $10 \mathrm{~m}$ to obtain similar counts. Figure 11 shows reconstructed ice crystal images from HOLODEC. The two small crystals are approximately $100 \mu \mathrm{m}$ in extent, for scale. This set of images makes it clear that HOLODEC is able to obtain cloud particle measurements spanning from cloud droplet to large ice crystal sizes, over large instantaneous sample volumes. 

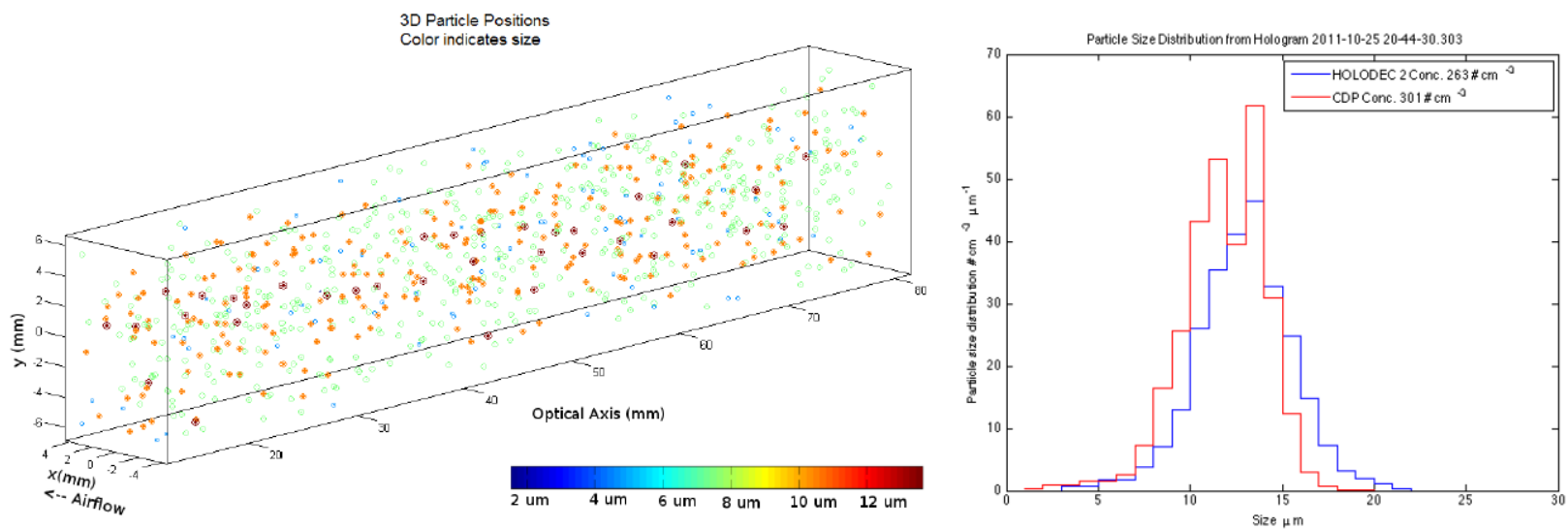

Figure 10. Full HOLODEC sample volume showing droplet spatial locations and droplet diameter (via color coding). The resulting size distribution is plotted to the right and is compared to one obtained from a CDP for a similar region of cloud.

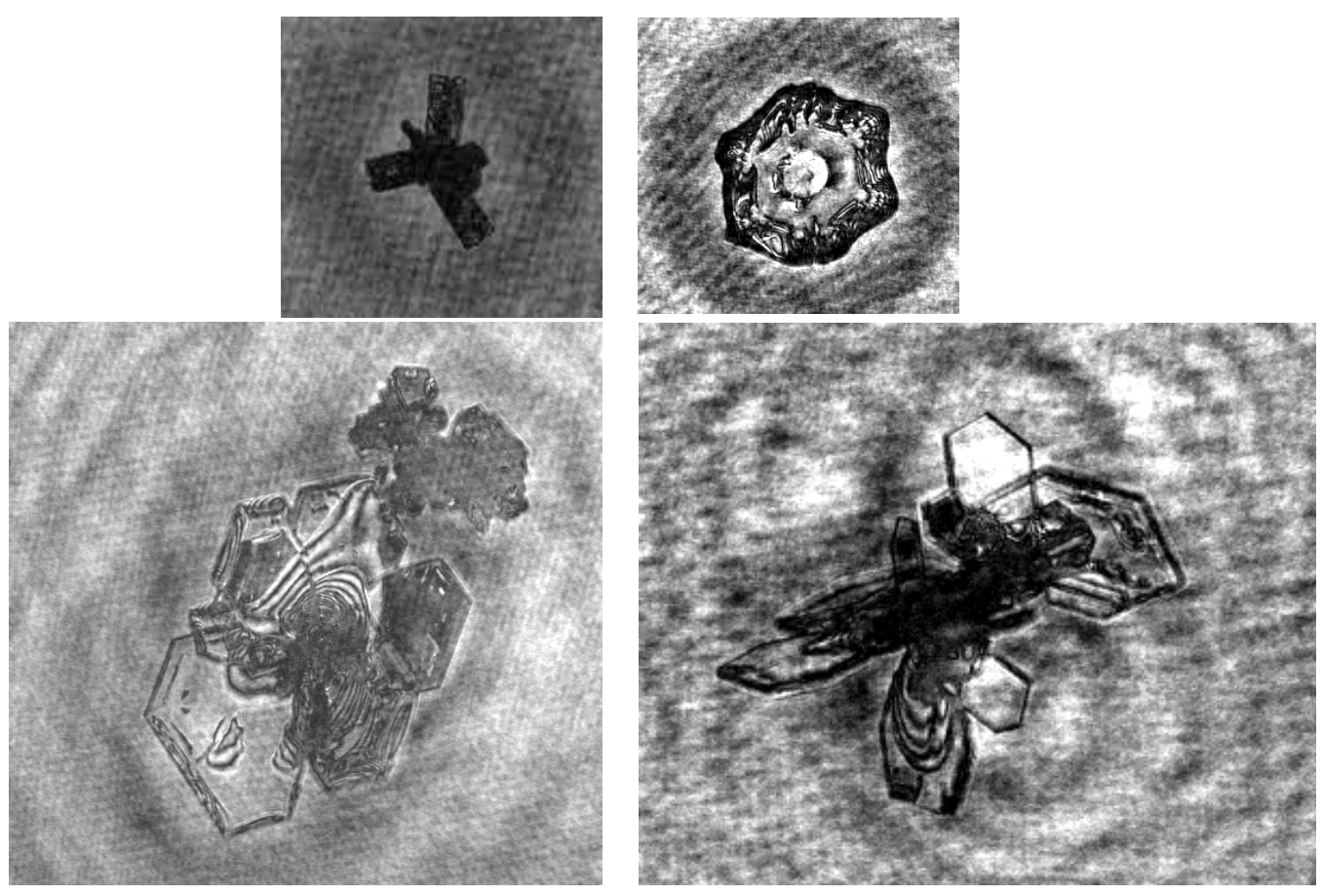

Figure 11. Reconstructed ice crystal images from HOLODEC. 


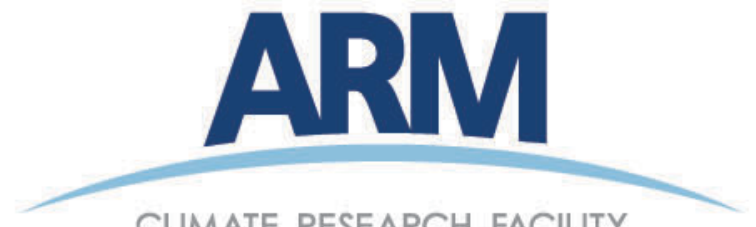

CLIMATE RESEARCH FACILITY

www.arm.gov

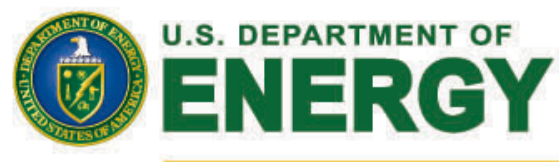

Office of Science 